\title{
PET Approaches for Diagnosis of Dementia
}

K. Ishii

\begin{abstract}
SUMMARY: There is increasing use of neuroimaging modalities, including PET, for diagnosing dementia. For example, FDG-PET demonstrates hypometabolic regions in the posterior cingulate gyri, precuneus, and parietotemporal association cortices, while amyloid PET indicates amyloid deposition in Alzheimer disease and mild cognitive impairment due to Alzheimer disease. Furthermore, the use of combination PET with structural MR imaging can improve the diagnostic accuracy of dementia. In other neurodegenerative dementias, each disease exhibits a specific metabolic reduction pattern. In dementia with Lewy bodies, occipital glucose metabolism is decreased, while in frontotemporal dementia, frontal and anterior temporal metabolism is predominantly decreased. These FDG-PET findings and positive or negative amyloid deposits are important biomarkers for various neurodegenerative dementias.
\end{abstract}

ABBREVIATIONS: $\mathrm{A} \beta=$ amyloid $\beta$ peptide; $\mathrm{AD}=$ Alzheimer disease; $A P O E=$ apolipoprotein $E ; \mathrm{CBD}=$ corticobasal degeneration; $\mathrm{DLB}=$ dementia with Lewy bodies; $\mathrm{FTD}=$ frontotemporal dementia; $\mathrm{MCl}=$ mild cognitive impairment; $\mathrm{PD}=$ Parkinson disease; $\mathrm{PiB}=$ Pittsburgh compound $-\mathrm{B} ; \mathrm{PSP}=$ progressive supranuclear palsy; VBM = voxel-based morphometry

$\mathrm{T}$ he number of patients with dementia is progressively increasing worldwide. Dementia is a clinical diagnosis based on significant and persisting deficits of intellectual function, usually memory. Diagnostic imaging techniques for morphologic examinations (eg, CT and MR imaging) and for functional examinations (eg, SPECT and PET) are now used widely for supporting the diagnosis of dementia. Such imaging techniques can indicate the likely underlying pathology in a patient who meets the clinical criteria for dementia. In particular, amyloid imaging PET has been reported to show the pathophysiologic states of dementia. The PET radiopharmaceutical $\left[{ }^{18} \mathrm{~F}\right]$ fluorodeoxyglucose can detect regional glucose metabolism in patients with dementia and is the most widely available and useful biomarker for dementia diagnosis. However, to exclude several pathologies such as subdural hematoma, vascular dementia, normal pressure hydrocephalus, and brain tumors, clinical examination is first performed by using MR imaging because FDG-PET alone cannot detect these diseases. After this process, FDG-PET is then performed to detect early neu-

From the Neurocognitive Disorders Center, Kinki University Hospital, Osaka, Japan. This work was supported by JSPS KAKENHI grant number 50534103 and 21st Century Research and Development Incentive Wages at Kinki University.

Paper previously presented at: 98th Scientific Assembly and Meeting of the Radiological Society of North America, November 25-30, 2012; Chicago, Illinois.

Please address correspondence to Kazunari Ishii, MD, Department of Radiology, Kinki University Faculty of Medicines, 377-2 Ohnohigashi, Osakasayama, Osaka 589-8511, Japan; e-mail: kishii@hbhc.jp

- Indicates open access to non-subscribers at www.ajnr.org

http://dx.doi.org/10.3174/ajnr.A3695 rodegenerative dementia, differentiate neurodegenerative dementias, ${ }^{1}$ or suggest comorbidity of other neurodegenerative diseases. Herein, I review the various imaging approaches for dementia, with a particular focus on PET and new techniques or tracers.

\section{Alzheimer Disease}

Alzheimer disease (AD) is the most common dementia, which starts with impairment of memory followed by multiple domains of cognitive dysfunction. The 2011 criteria for $\mathrm{AD}^{2,3}$ was revised from that in $1984,{ }^{4}$ and the recommendations regarding the respective roles of FDG-PET and MR imaging in AD and mild cognitive impairment (MCI) are conflicting. However, PET plays an important role as a biomarker in $\mathrm{AD}$, as well as for functional and molecular imaging. For example, FDG-PET is described as a "neuronal injury" biomarker in $\mathrm{AD} .^{2,3}$

\section{FDG-PET in Alzheimer Disease}

FDG-PET can demonstrate a glucose metabolic reduction in the parietotemporal association cortices, posterior cingulate, and precuneus regions at the early stage of $\mathrm{AD}$ (Fig 1). By contrast, in the healthy elderly brain, posterior cingulate glucose metabolism is much higher than that in other cortices, while parietal glucose metabolism is the same as that in the primary sensorimotor cortices (Fig 2 ). In the moderate-to-severe stages of $\mathrm{AD}$, hypometabolic regions spread to the frontal association cortices, while metabolism in the striatum, thalamus, primary sensorimotor cortices, visual cortices, and cerebellum are relatively preserved despite disease progression.

Because the magnitude of glucose metabolic reduction in the medial temporal lobe, including the hippocampus, is not as large 


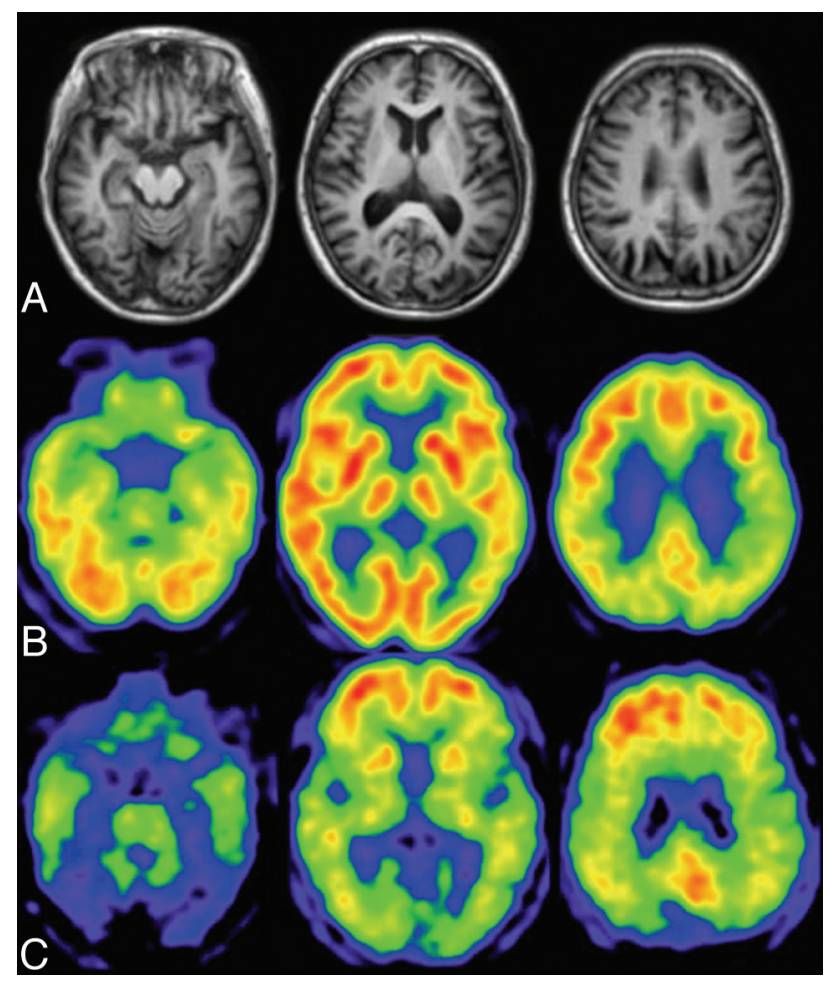

FIG 1. A patient with early Alzheimer disease, 77 years of age, MiniMental State Examination score $=25$. $A$, Minimal atrophy was seen in the right hippocampus. $B$, FDG-PET shows reduced glucose metabolism in the bilateral parietotemporal association cortices and posterior cingulate gyri and precuneus. $\mathrm{C}$, PiB accumulations are demonstrated in the cerebral cortices except for the occipital and medial temporal regions. Medial parietal and frontal accumulations of PiB are high, indicative of positive amyloid deposit.

as that in the parietotemporal or posterior cingulate cortices, a decrease in hippocampal glucose metabolism with progression from mild-to-moderate-stage $\mathrm{AD}$ is not usually demonstrated, ${ }^{5}$ despite obvious hippocampal atrophy at the early stage. Hippocampal glucose metabolism is somewhat decreased at the early stage of AD. However, it is difficult to detect the small reduction of hippocampal glucose metabolism in patients with early $\mathrm{AD}$ because the hippocampal glucose metabolism is smaller than the neocortical glucose metabolism, even in healthy subjects, and the magnitude of the decrease is modest. Thus, a method for detecting hippocampal metabolic reduction at an early stage of $\mathrm{AD}$ is required..$^{6-8}$ However, Karow et $\mathrm{al}^{9}$ reported that FDG-PET has the same sensitivity as MR imaging for detecting brain degeneration in preclinical and mild $\mathrm{AD}$, suggesting that $\mathrm{MR}$ imaging may be a more practical clinical biomarker for early detection of $\mathrm{AD}$.

\section{Mild Cognitive Impairment Due to Alzheimer Disease}

Mild cognitive impairment consists of a heterogeneous pathology, and $\mathrm{MCI}$ due to $\mathrm{AD}$ is a transitional stage between aging and $\mathrm{AD}$. MCI due to $\mathrm{AD}$ demonstrates the same glucose-reduction pattern as early $\mathrm{AD}$ (Fig 3 ), which predicts that the patient will show symptoms of $\mathrm{AD}$ in the near future. At the early stage of $\mathrm{AD}$ or MCI due to $\mathrm{AD}$, it is difficult to detect the characteristic hypometabolic patterns on FDG-PET images by visual inspection. As such, statistical images are helpful. Nevertheless, FDG-PET generally has a higher accuracy than $\mathrm{MR}$ imaging for diagnosing early $\mathrm{AD}^{10,11}$ and

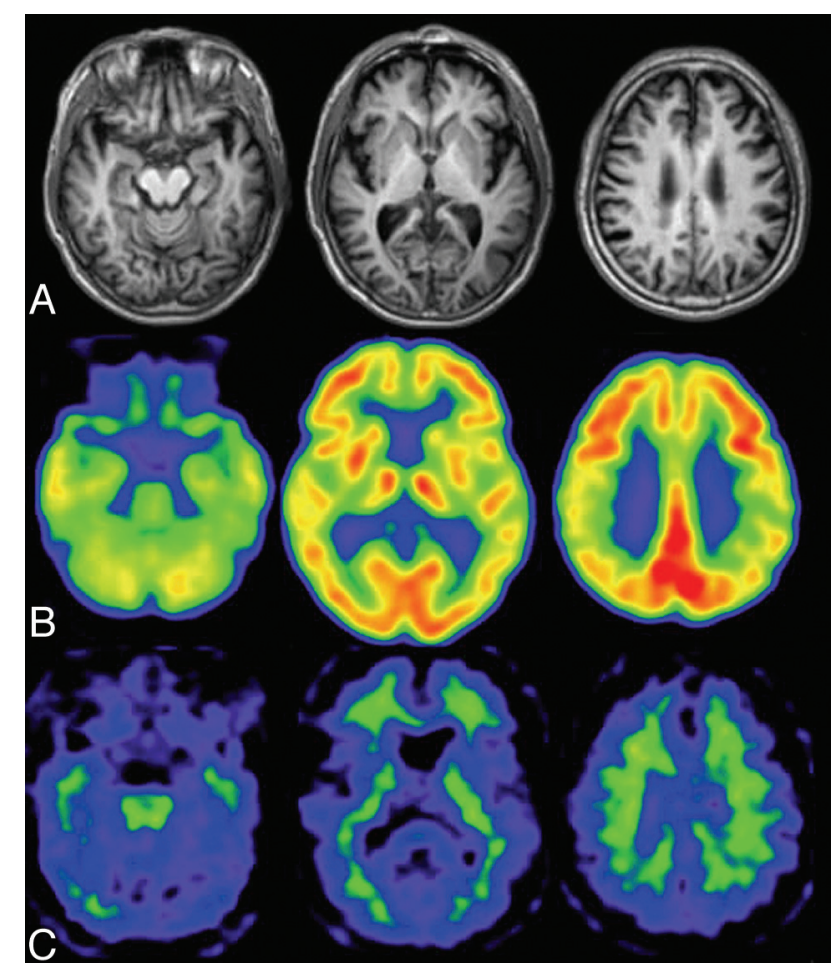

FIG 2. Healthy elderly male subject, 78 years of age, Mini-Mental State Examination score $=30$. A, A slight enlargement of the right inferior horn of the lateral ventricle is seen on the Tl-weighted MR image. $B$, The regional glucose metabolism is not reduced on the FDG-PET images. Note that the posterior cingulate glucose metabolism is much larger than that in other regions. C, PiB-PET shows nonspecific accumulation in the white matter but no PiB accumulation in the gray matter. The amyloid deposit is negative.

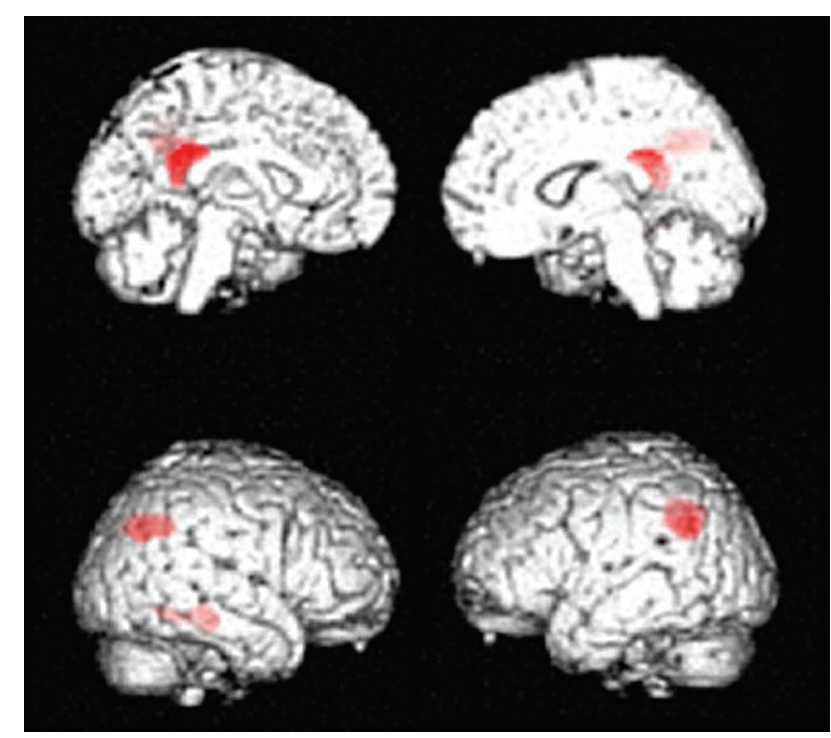

FIG 3. $M C l$ due to $A D$. Regions exhibiting a significant reduction in glucose metabolism in patients with $\mathrm{MCl}$ due to $\mathrm{AD}(n=20) \mathrm{com}$ pared with healthy elderly subjects $(n=20)$ are demonstrated by statistical parametric maps. Bilateral parietal and posterior cingulate metabolism is decreased in patients with $\mathrm{MCl}$ due to AD. These decreased regions are the same as those in patients with early $A D$.

for predicting rapid conversion of $\mathrm{MCI}$ to early $\mathrm{AD} .{ }^{12} \mathrm{~A}$ combination of PET and other biomarkers is important because imaging and CSF biomarkers can improve prediction of conversion from $\mathrm{MCI}$ to $\mathrm{AD}$ AJNR Am J Neuroradiol 35:2030-38 Nov 2014 www.ajnr.org 


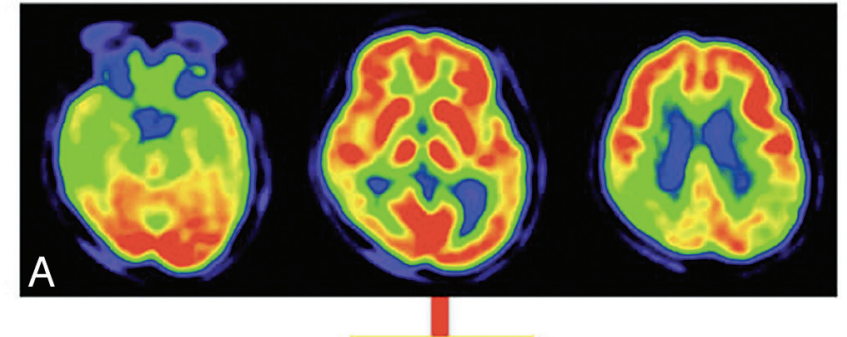

\section{D-SSP}

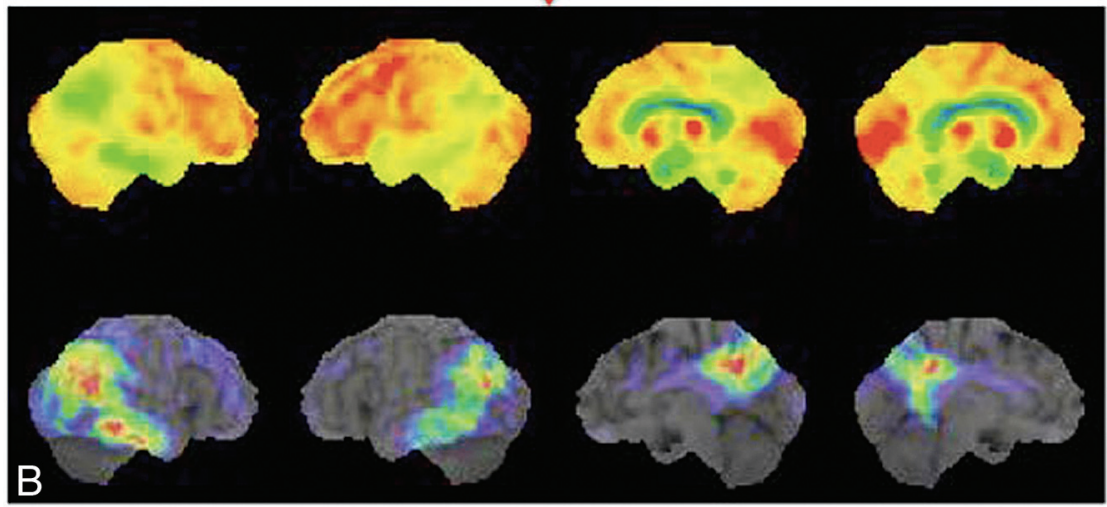

FIG 4. Process of voxel-based statistical maps. Original FDG-PET image of a patient with mild AD $(A)$ is analyzed by using the 3D-SSP program, and the 3D-SSP surface map and $z$ score map are produced. The $z$ score map shows the regions with significantly reduced metabolism compared with a normal data base $(B)$, which aid in the diagnosis of $A D$.

compared with baseline clinical testing. FDG-PET appears to add the greatest prognostic information. ${ }^{13-16}$

\section{Effects of APOE on FDG-PET and Amyloid Imaging of Alzheimer Disease}

The apolipoprotein $E$ gene (APOE) is a risk factor for late-onset $\mathrm{AD}$, while the $A P O E \varepsilon 4$ allele $(\varepsilon 4)$ increases the risk and decreases the average age of dementia onset. Patients with early-onset $\mathrm{AD}$ who were APOE $\varepsilon 4$-positive showed a significant decrease in glucose metabolism in the medial temporal lobe compared with patients with $A P O E \varepsilon 4-$ negative $\mathrm{AD} .{ }^{17}$ By contrast, in the late-onset group, there were no differences in the glucose-metabolic reduction patterns between patients with $A P O E \varepsilon 4$-positive and-negative AD. A similar pattern of cerebral hypometabolism was also detected between patients with $A P O E \varepsilon 4$-positive and -negative AD but with stronger abnormalities in $\varepsilon 4$ carriers in the parietal, temporal, and posterior cingulate cortical regions. ${ }^{18}$ Recently Ossenkoppele et $\mathrm{al}^{19}$ found a reversed APOE $\varepsilon 4$ dose effect for amyloid deposition in the frontal lobe, whereas APOE $\varepsilon 4$ carriership was associated with more profound metabolic impairment in the posterior parts of the cortex. These findings suggest that the APOE genotype has a differential effect on the distribution of amyloid plaques and glucose metabolism.

\section{Statistical Voxel-Based Analysis Approach for Alzheimer Disease Diagnosis}

Minoshima et $\mathrm{al}^{20,21}$ first developed the 3D stereotactic surface projection system (3D-SSP; http://www.rad.washington.edu/research/
Research/groups/nbl/neurostat-3d-ssp) and used $z$ score images to aid visual inspection of individual FDG images for diagnosing $\mathrm{AD}$ (Fig 4). The Statistical Parametric Mapping program (Wellcome Department of Imaging Neuroscience, London, UK) was developed as a tool for analysis of PET and functional MR imaging images. For automatic diagnosis, Herholz et $\mathrm{al}^{22}$ proposed the $t$-sum method of calculating the total $t$-values in a region-of-interest template by using the Statistical Parametric Mapping program; by using this method, the parietotemporal and frontal regions were found to be $\mathrm{AD}$ specific compared with healthy elderly subjects. An automatic diagnosis system by using 3D-SSP and a region-of-interest template was proposed for discriminating those with early $\mathrm{AD}$ from healthy subjects. $^{23,24}$ This system was also applied to distinguish patients with dementia with Lewy bodies (DLB) from those with $\mathrm{AD} .^{24}$ All these systems require a data base of healthy controls. More recently, however, a data base-independent system was proposed by using $t$-statistics between parietal and sensorimotor glucose metabolism in individual subjects. ${ }^{25}$ Mevel et $\mathrm{al}^{26}$ proposed an automatic system for determining hippocampal metabolism by using a voxel-based method and partial volume correction, which was useful in detecting MCI.

\section{Combination of MR Imaging and PET}

Most of the studies described above have focused on only a single technique such as structural MR imaging or PET. Yuan et $\mathrm{al}^{12}$ performed a meta-analysis and meta-regression on the diagnostic performance data for MR imaging, SPECT, and FDG-PET in subjects with MCI and reported that FDG-PET performed slightly better than SPECT and structural MR imaging in the prediction of conversion to $\mathrm{AD}$ in patients with $\mathrm{MCI}$, while a combination of PET and structural MR imaging improved the diagnostic accuracy of dementia. Kawachi et $\mathrm{al}^{10}$ also compared the diagnostic performance of FDG-PET and voxel-based morphometry (VBM) on MR imaging in the same group of patients with very mild $\mathrm{AD}$ and reported an accuracy of $89 \%$ for FDG-PET diagnosis and $83 \%$ for VBM-MR imaging diagnosis, while the accuracy of combination FDG-PET and VBM-MR imaging diagnosis was $94 \%$. These studies suggest that a combination of imaging modalities may improve the diagnosis of $\mathrm{AD}$.

\section{Amyloid Imaging in Alzheimer Disease}

The most widely used tracer for amyloid imaging is ${ }^{11} \mathrm{C}$-Pittsburgh compound-B (PiB), ${ }^{27}$ which has a high affinity to amyloid $\beta$ peptide $\left(\mathrm{A} \beta\right.$ ) aggregates. The ${ }^{11} \mathrm{C}-\mathrm{BF} 227$ ligand is also used for amyloid imaging, ${ }^{28}$ though its uptake contrast between amyloidpositive and -negative brain tissue is not as high as that for $\mathrm{PiB}$, and it is used only at a few institutions in Japan. Because the 
Table 1: Representative radiopharmaceuticals for amyloid imaging

\begin{tabular}{|c|c|}
\hline${ }^{11} \mathrm{C}-\mathrm{PiB}$ & {$\left[{ }^{11} \mathrm{C}\right]-2-4-\left(\right.$ methylaminophenyl)- 6-hydroxybenzothiazole ${ }^{62}$} \\
\hline${ }^{11} \mathrm{C}-\mathrm{BF} 227$ & {$\left[{ }^{11} \mathrm{C}\right] 2-\left[2-\left(2-\right.\right.$ Dimethylaminothiazol-5-yl)ethenyl-6-[2-(fluoro)ethoxy]benzoxazole ${ }^{28}$} \\
\hline${ }^{11 C-A Z D 2184}$ & $\mathrm{~N}-\left[{ }^{[1]} \mathrm{C}\right]$ methyl)-2-(6-methylamino-pyridine-3-yl)-benzo[d]thiazole-6-ol ${ }^{66}$ \\
\hline${ }^{18} \mathrm{~F}-\mathrm{FDDNP}$ & 2-(1-(6-[(2-[18 F]fluoroethyl)(methyl)amino]-2-naphthyl)ethylidene)malononitrile ${ }^{67}$ \\
\hline${ }^{18} \mathrm{~F}-\mathrm{PiB}$ & $2-\left[3-\left[{ }^{8} \mathrm{~F}\right]\right.$ fluoro-4-(methylamino)phenyl]1,3-benzothiazole-6-ol ${ }^{68}$ \\
\hline${ }^{18} \mathrm{~F}-\mathrm{AV} 45$ & (E)-4-(2-(6-(2-(2-(2-[ $\left.{ }^{18} \mathrm{~F}\right]-$ fluoroethoxy)ethoxy)ethoxy)pyridin-3-yl)vinyl)-N-methyl benzenamine ${ }^{30}$ \\
\hline${ }^{18} \mathrm{~F}-\mathrm{BAY} 94-9172$ & 4-(N-methylamino)-4'-(2-(2-(2-[18 F]fluoroethoxy)-ethoxy)-ethoxy)-stilbene ${ }^{69}$ \\
\hline${ }^{18} \mathrm{~F}-\mathrm{AZD} 4694$ & 2-(2-[ ${ }^{18}$ F]fluoro-6-(methylamino)pyridin-3-yl)benzofuran-5-ol ${ }^{30}$ \\
\hline
\end{tabular}

Note:- ${ }^{18}$ F-AV45 indicates florbetapir; ${ }^{18} \mathrm{~F}-\mathrm{BAY} 94-9172$, florbetaben; ${ }^{18} \mathrm{~F}-\mathrm{PiB}$, flutemetamol.

half-life of the ${ }^{11} \mathrm{C}$ isotope is short, ${ }^{11} \mathrm{C}-\mathrm{PiB}$ is available only in specific institutions that have a cyclotron and a synthesis system. Conversely, the half-life of the ${ }^{18} \mathrm{~F}$ isotope is 110 minutes, and ${ }^{18} \mathrm{~F}$-labeled amyloid imaging tracers will become widely used following production by radiopharmaceutical companies. Currently there are a few ${ }^{18} \mathrm{~F}$-labeled amyloid imaging tracers available ( $\mathrm{Ta}$ ble 1), ${ }^{29}$ though in $2012,{ }^{18} \mathrm{~F}-\mathrm{AV}-45$ (florbetapir) ${ }^{30}$ was approved by the FDA for PET imaging of $\beta$-amyloid neuritic plaques in the living brain. ${ }^{18} \mathrm{~F}$-AZD4694 is also expected to be a good radioligand for both diagnostic use and evaluation of disease-modifying therapies in $\mathrm{AD} .^{31}$

Amyloid PET is helpful in the diagnosis of AD. However, the timing of amyloid accumulation is at a preclinical stage of $\mathrm{AD} .{ }^{32}$ Early $\mathrm{A} \beta$ accumulation and increased vulnerability to $\mathrm{A} \beta$ pathology play critical roles in the pathogenesis of patients with earlyonset $\mathrm{AD} .{ }^{33} \mathrm{~A} \beta$ deposition initiates the pathologic cascade but is not the direct cause of cognitive impairment, and intermediate $\mathrm{A} \beta$ deposit may represent individuals with $\mathrm{AD}$ coexistent with other pathologies. ${ }^{34}$ Furthermore, amyloid deposit is not unique to $\mathrm{AD}$ and can be found in other diseases or healthy elderly people.

At present, amyloid PET is not indicated in patients with preclinical AD or healthy subjects, except for clinical trials or research studies. The appropriate usage criteria for amyloid PET were proposed by the Amyloid Imaging Task Force, the Society of Nuclear Medicine and Molecular Imaging, and the Alzheimer's Association. ${ }^{35,36}$ These criteria indicate that appropriate use of amyloid PET includes the following: 1) patients with persistent or progressive unexplained MCI; 2) patients with possible AD with unclear clinical presentation, either an atypical clinical course or an etiologically mixed presentation; and 3) patients with progressive dementia and atypically early age of onset. Inappropriate use of amyloid PET includes the following: 1) in patients with typical probable $\mathrm{AD}, 2$ ) for determining the severity of dementia, 3) in subjects with a positive family history of dementia or the presence of $A P O E \varepsilon 4,4)$ in patients with a cognitive problem that is unconfirmed on clinical examination, 5) as a replacement for genotyping for suspected autosomal mutation carriers, 6) in asymptomatic subjects, and 7) for nonmedical use (eg, legal, insurance coverage, or employment screening).

\section{Dementia with Lewy Bodies}

Dementia with Lewy bodies is the second most common neurodegenerative dementia following $\mathrm{AD}$ and is characterized pathologically by the presence of Lewy bodies in cortical, subcortical, and brain stem structures. DLB belongs to the spectrum of Lewy body diseases including Parkinson disease (PD), where Lewy bodies remain in the brain stem. PD with dementia has the same

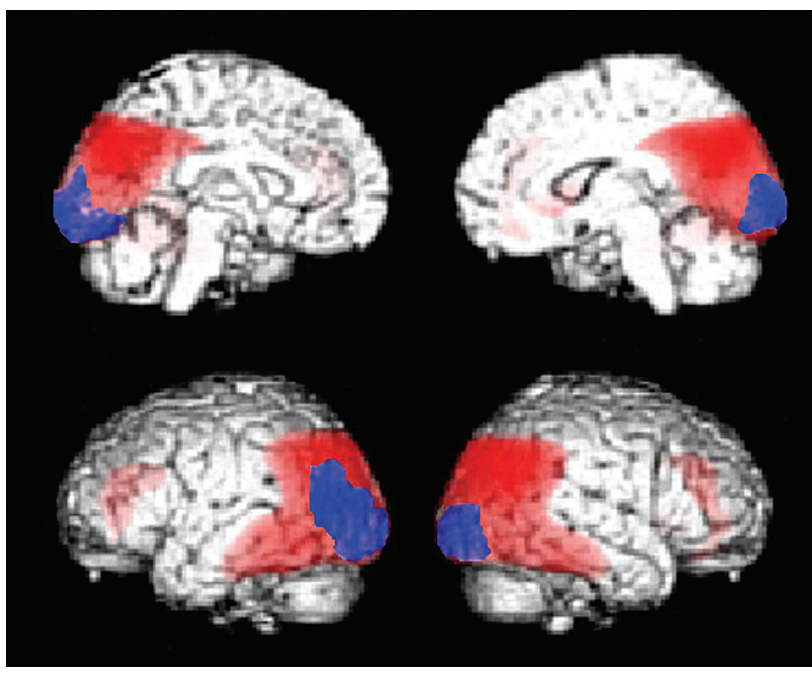

FIG 5. Decreased glucose metabolic regions of DLB $(n=20)$. Statistical parametric maps show the areas where glucose metabolism is significantly decreased compared with age-matched healthy controls $(n=20)$. The red area indicates the parietotemporal association area, and the posterior cingulate gyri overlap the area where glucose metabolism is decreased in AD. The blue area indicates occipital cortices that are specific for DLB, where the glucose metabolism is preserved in $\operatorname{AD}(n=20)$.

pathology as DLB, though clinical symptoms slightly differ: Parkinsonism appears $>1$ year earlier than cognitive dysfunctions in $\mathrm{PD}$ with dementia.

In 2005, the criteria for clinical and pathologic diagnoses were revised $^{36}$ and included an important role for functional and morphologic imaging. For example, low dopamine transporter uptake in the basal ganglia on SPECT and PET imaging scans is regarded as a suggestive feature for DLB, while supportive features include relative preservation of medial temporal lobe structures on $\mathrm{CT} / \mathrm{MR}$ imaging and generalized low uptake on SPECT/PET, with reduced occipital activity and abnormal (low) uptake on metaiodobenzylguanidine myocardial scintigraphy. Relative preservation of the hippocampus in DLB compared with prominent hippocampal atrophy in $\mathrm{AD}$ is also a common finding, though there are many overlapping areas of hippocampal atrophy between patients with DLB and those with $\mathrm{AD}$, making it impossible to distinguish a patient with DLB from one with AD by using only a finding of hippocampal atrophy.

The hypometabolic regions in patients with DLB are similar to those in $\mathrm{AD}$, though patients with DLB exhibit a difference in the involved occipital lobe (Fig 5). The relative metabolic reduction was more severe in the occipital cortices and less severe in the medial temporal lobes in DLB compared with $\mathrm{AD}$, and occipital hypometabolism is a key feature of DLB that discriminates it from 


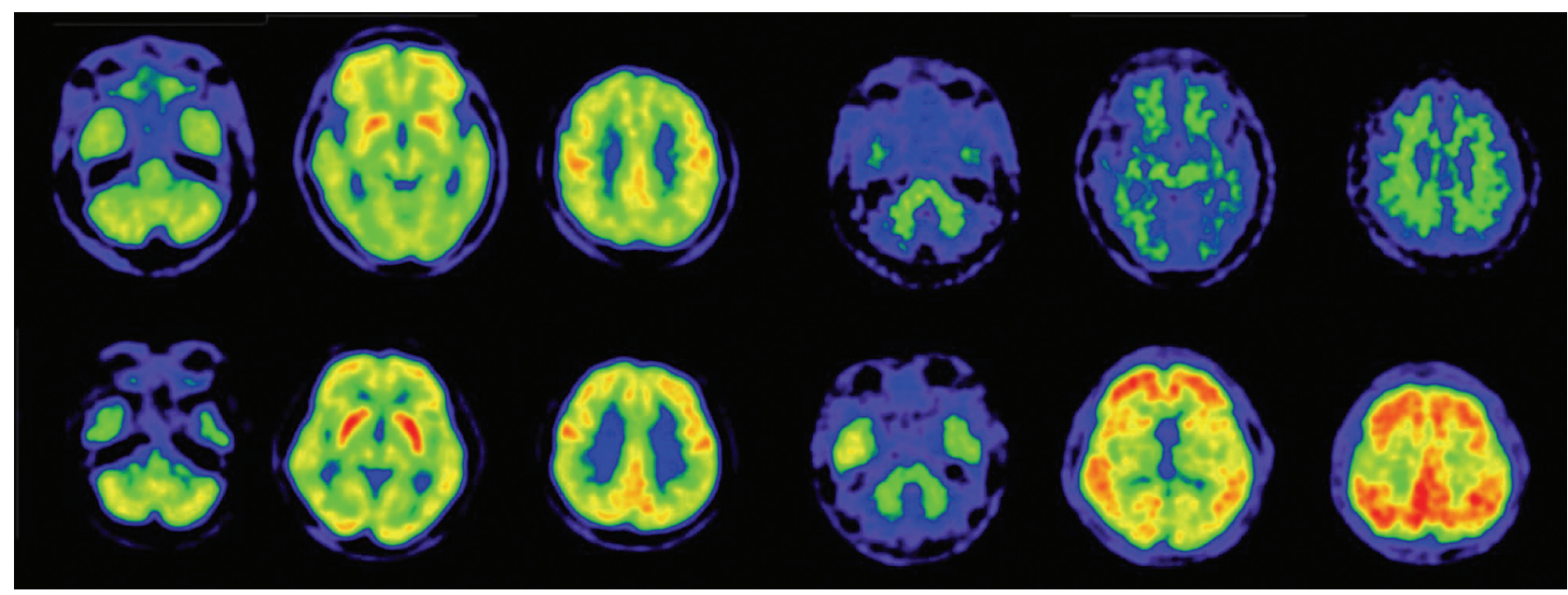

FIG 6. PiB-PET and FDG-PET images of patients with DLB. Upper row shows a 76-year-old man with DLB. This patient has Parkinsonism and cognitive fluctuation. His Mini-Mental State Examination score was 19. Diffuse glucose metabolic reduction is demonstrated in all regions apart from the striatum and primary sensorimotor cortices (upper left). PiB-PET (upper right) demonstrates a negative amyloid deposit. Lower row shows a 77-year-old woman with DLB, Parkinsonism, and cognitive fluctuation. Her Mini-Mental State Examination score was 23. FDG-PET demonstrates decreased glucose metabolism in the parietotemporal and frontal association cortices, occipital cortices, and posterior cingulate gyri, while metabolism in the striatum and primary sensorimotor metabolism is spared (lower left). PiB-PET demonstrated diffuse amyloid deposition in the cerebral cortices (lower right).

AD. ${ }^{7,38,39}$ Nevertheless, pathologically, the occipital cortices are not as involved in patients with DLB. These findings are also seen in patients with PD and PD with dementia ${ }^{40}$ because DLB and PD with dementia are in the same disease entity, involving spread of Lewy bodies in the cerebral cortices.

Dopamine transporter imaging with ${ }^{123} \mathrm{I}-\mathrm{N}$ - $\omega$-fluoropropyl$2 \beta$-carbomethoxy-3 $\beta$-(4-iodophenyl)nortropane ([ $\left.{ }^{123} \mathrm{I}\right] \mathrm{FP}$-CIT) SPECT imaging is useful for diagnosing DLB and exhibits a diagnostic accuracy sufficient to distinguish DLB from AD. ${ }^{41}$ However, this imaging technique cannot distinguish other degenerative dementias with Parkinsonism (eg, progressive supranuclear palsy (PSP) and multiple system atrophy), while FDG-PET imaging can distinguish DLB from PSP and multiple-system atrophy. ${ }^{123}$ I-metaiodobenzylguanidine cardiac scintigraphy, which is typically used for myocardial innervation, also demonstrates a high sensitivity and specificity in patients with suspected DLB and provides an accurate examination for differential diagnosis between DLB and other dementias. $^{42}$

\section{Amyloid Imaging in Dementia with Lewy Bodies}

Shimada et $\mathrm{al}^{43}$ reported that amyloid deposits are associated with AD-like atrophy in patients with DLB/PD with dementia. Patients with DLB have higher amyloid deposits than patients with PD and PD with dementia. Amyloid deposits have been linked to cognitive impairment in $\mathrm{DLB}^{44,45}$ and may contribute to the timing of the onset of dementia relative to that of Parkinsonism in Lewy body dementia. ${ }^{46}$ However, as shown in Fig 6, severe metabolic reduction was seen in some patients with DLB despite no evidence of amyloid deposits. In particular, regional metabolic reduction in patients with DLB was observed in the parietotemporal, posterior cingulate, and frontal association cortices, which are the same regions affected in $\mathrm{AD}$ but are not correlated with amyloid deposit.

\section{Frontotemporal Lobe Degeneration}

Frontotemporal lobe degeneration (FTD) is a heterogeneous group of diseases involving frontal and/or anterior temporal lobe

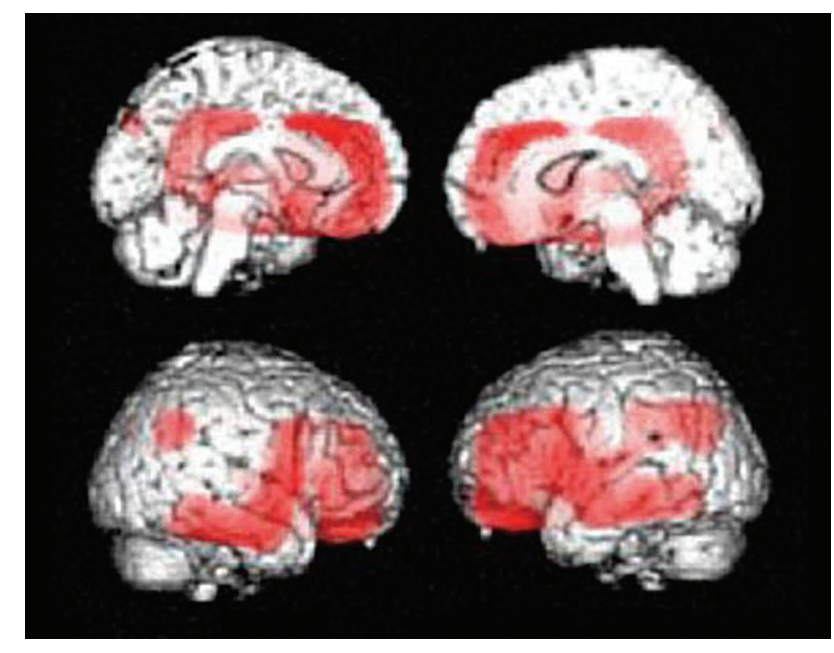

FIG 7. Hypometabolic regions in FTD. Statistical parametric mapping analysis shows hypometabolic regions in patients with FTD $(n=14)$ compared with healthy age-matched subjects $(n=20)$. Glucose metabolism in subjects with FTD was significantly decreased in the bilateral lateral and medial frontal cortices, the posterior cingulate gyrus, and small regions in the parietal association cortices.

degeneration and associated dementia. Frontotemporal dementia is a comprehensive clinical entity of primary degenerative dementia characterized by peculiar behavioral changes and includes 3 types of histologic change (pick-type, frontal lobe degeneration type, and motor neuron disease type). ${ }^{47}$ Frontotemporal lobe degeneration includes FTD, semantic dementia, and progressive nonfluent aphasia, while FTD is now used clinically to classify patients with frontal and temporal symptoms.

In FTD, frontal and anterior temporal glucose metabolism is decreased, though the medial temporal region and the subcortical structures, including the striatum and thalamus, are also affected (Fig 7). ${ }^{48}$ According to a VBM study of FTD,${ }^{49}$ metabolic and atrophic changes occur in the bilateral frontal and temporal lobes, 


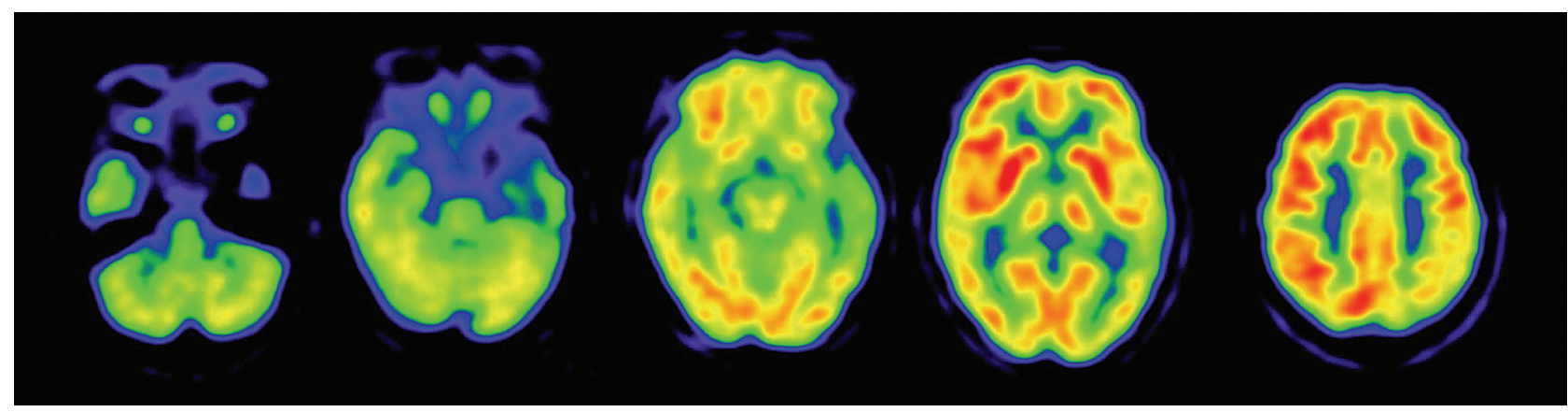

FIG 8. FDG-PET image of a 54-year-old female patient with semantic dementia. A marked decreased left anterior temporal metabolism is shown on the FDG-PET image.

whereas the affected regions of metabolism are larger and more severe than those of atrophy in the frontal lobe. In patients with semantic dementia, asymmetrically severely decreased temporal metabolism can be demonstrated on FDG-PET images (Fig 8). These areas can spread to the frontal and parietal cortices, though the degree of metabolism alteration is not as marked as in the anterior temporal cortices.

\section{Amyloid Imaging in Frontotemporal Lobe Degeneration} In a study of PiB retention in patients with FTD, Engler et $\mathrm{al}^{50}$ reported that most patients with FTD displayed no $\mathrm{PiB}$ retention and that PiB could potentially aid in differentiating FTD and AD. Rabinovici et $\mathrm{al}^{51}$ also reported that $8 / 12$ patients with frontotemporal lobe degeneration had negative amyloid deposits, and the 4 PiB-positive patients with frontotemporal lobe degeneration may suggest the mimicking of AD pathology.

\section{Progressive Supranuclear Palsy}

Progressive supranuclear palsy is a neurotauopathic degenerative disorder presenting with Parkinsonism and dementia. The neurologic features include impaired ocular motility, pseudobulbar palsy, and axial dystonia. The basal ganglia and brain stem are the main loci of pathologic involvement. MR imaging studies demonstrate brain stem atrophy with a so-called "humming bird" sign, though these findings are not often seen at the early stage of PSP. However, FDG-PET demonstrates decreased glucose metabolism in the midbrain, the lateral and medial frontal lobes, and the caudate nucleus, even at the early stage of PSP (Fig 9) ${ }^{52,53}$ and can distinguish PSP from other diseases with Parkinsonism. ${ }^{54,55}$

\section{Corticobasal Degeneration}

Corticobasal degeneration (CBD), which belongs to the corticobasal syndrome, is characterized by akineto-rigid syndrome, dystonia, myoclonus, apraxia, alien limb syndrome, and cortical sensory loss, and typically occurs with asymmetric onset. CBD is pathologically a tauopathic neurodegenerative disease and includes $\tau$-immunoreactive neuronal cytoplasmic inclusions, oligodendroglial inclusions, and astrocytic plaques. FDG-PET demonstrates hypometabolism in the frontal and parietal cortices and the subcortical structures, including the thalamus, caudate nucleus, and putamen, with marked asymmetry between the 2 hemispheres. Patients with CBD with symptoms of dementia show an AD-like metabolic reduction pattern but asymmetric glucosemetabolic reduction in the lateral frontal, lateral temporal, medial

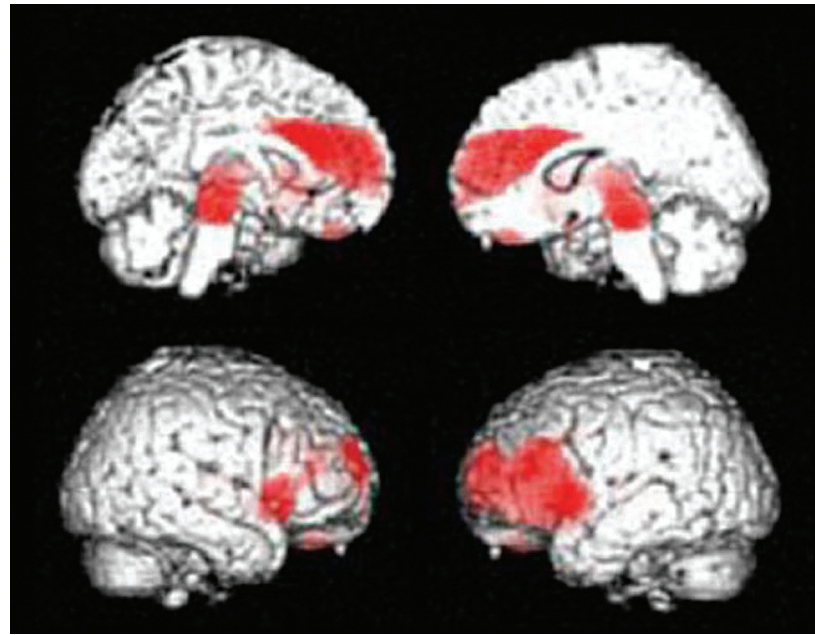

FIG 9. Hypometabolic regions in PSP. Statistical parametric mapping analysis shows hypometabolic regions in patients with PSP $(n=16)$ compared with normal age-matched subjects $(n=20)$. Glucose metabolism in subjects with PSP is significantly decreased in the bilateral lateral and medial frontal cortices and midbrain. The significant decreased midbrain metabolism is a hallmark of PSP.

and lateral parietal cortices; the pre- and postcentral gyri; and the thalamus on FDG-PET. ${ }^{56}$

\section{Amyloid Imaging in Progressive Supranuclear Palsy and Corticobasal Degeneration}

PSP and CBD are tauopathic neurodegenerative diseases that exhibit no amyloid deposition. If amyloid deposition is demonstrated with amyloid PET, it should be considered small amyloid deposits with age-related amyloid pathology or extended amyloid deposits similar to those in $\mathrm{AD}$ due to comorbidity of $\mathrm{AD}$, which may be associated with $A P O E \varepsilon 4$.

\section{Idiopathic Normal Pressure Hydrocephalus}

Idiopathic normal pressure hydrocephalus is characterized by disproportionately enlarged cerebrospinal spaces, ventricle and Sylvian fissure dilation, and tight sulci at high convexity in the medial parietal sulci. ${ }^{57}$ To detect these features, morphologic images by using CT or MR imaging are necessary. On the other hand, functional images are not necessary because the glucose metabolic reduction pattern is heterogeneous in idiopathic normal pressure hydrocephalus. ${ }^{58}$ Glucose metabolism in idiopathic normal pressure hydrocephalus before and after a 
Table 2: General FDG-PET and amyloid PET findings in neurodegenerative dementia

\begin{tabular}{|c|c|c|c|c|c|c|c|c|c|c|c|}
\hline & PC & Pari & Lat. Temp & Med. Temp & Fron & Occ & SM & ST & TH & BS & AMY \\
\hline $\mathrm{MCl}$ due to $\mathrm{AD}$ & $\downarrow$ & $\downarrow$ & $\rightarrow$ & $\rightarrow$ (or $\downarrow$ ) & $\rightarrow$ & $\rightarrow$ & $\rightarrow$ & $\rightarrow$ & $\rightarrow$ & $\rightarrow$ & + or ++ \\
\hline AD (mild) & $\downarrow$ & $\downarrow$ & $\downarrow$ & $\rightarrow$ (or $\downarrow$ ) & $\rightarrow$ & $\rightarrow$ & $\rightarrow$ & $\rightarrow$ & $\rightarrow$ & $\rightarrow$ & ++ \\
\hline AD (moderate) & $\downarrow$ & $\downarrow$ & $\downarrow$ & $\downarrow$ & $\downarrow$ & $\rightarrow$ & $\rightarrow$ & $\rightarrow$ & $\rightarrow$ & $\rightarrow$ & ++ \\
\hline DLB & $\downarrow$ & $\downarrow$ & $\downarrow$ & $\rightarrow$ & $\downarrow$ & $\downarrow$ & $\rightarrow$ & $\rightarrow$ & $\rightarrow$ & $\rightarrow$ & + or - \\
\hline FTD & $\rightarrow$ (or $\downarrow)$ & $\rightarrow$ (or $\downarrow)$ & $\downarrow$ & $\downarrow$ & $\rightarrow$ & $\rightarrow$ & $\rightarrow$ & $\rightarrow$ or $\downarrow$ & $\rightarrow$ & $\rightarrow$ & - \\
\hline SD & $\rightarrow$ & $\rightarrow$ (or $\downarrow)$ & $\downarrow \downarrow$ & $\downarrow$ & $\rightarrow$ & $\rightarrow$ & $\rightarrow$ & $\rightarrow$ & $\rightarrow$ & $\rightarrow$ & - \\
\hline PSP & $\rightarrow$ & $\rightarrow$ & $\rightarrow($ or $\downarrow)$ & $\rightarrow$ & $\downarrow$ & $\rightarrow$ & $\rightarrow$ & $\downarrow$ & $\rightarrow$ & $\downarrow$ & - \\
\hline CBD 1) & $\downarrow$ & $\downarrow$ & $\downarrow$ & $\rightarrow$ & $\downarrow$ & $\rightarrow$ & $\downarrow$ & $\downarrow$ & $\downarrow$ & $\rightarrow$ & - \\
\hline VaD 2) & $\rightarrow$ (or $\downarrow)$ & $\rightarrow$ (or $\downarrow$ ) & $\rightarrow$ or $\downarrow$ & $\rightarrow$ or $\downarrow$ & $\downarrow$ & $\rightarrow$ & $\rightarrow$ or $\downarrow$ & $\rightarrow$ or $\downarrow$ & $\rightarrow$ or $\downarrow$ & $\rightarrow$ & - \\
\hline
\end{tabular}

Note:-PC indicates posterior cingulate gyrus; Pari, parietal association cortex; Lat. Temp, lateral temporal cortex; Med. Temp, medial temporal cortex; Fron, frontal cortex; Occ, occipital cortex; SM, sensorimotor cortex; ST, striatum; TH, thalamus; BS, brain stem; AMY, amyloid deposition: 1) asymmetric reduction is characteristic, 2) frontal dominant metabolic reduction is often demonstrated in addition to infarction region; SD, semantic dementia; VaD, vascular dementia; $\downarrow$, decreased; $\rightarrow$, preserved; + , positive; -, negative.

shunt operation is promptly reversible after surgery, and there is a relationship between the early metabolic changes and clinical symptoms that is independent of the simultaneous changes in ventricular size. ${ }^{59}$ Because idiopathic normal pressure hydrocephalus occurs in elderly individuals, there is a possibility for concomitant AD. Amyloid imaging with PET may be useful to detect early concomitant $\mathrm{AD}$ and other diseases associated with amyloid deposits. ${ }^{60}$

\section{Vascular Dementia}

Vascular dementia is diagnosed with clinical symptoms and by detecting symptoms related to vascular lesions demonstrated by morphologic imaging. As such, pure vascular dementia is not a PET-applicable dementia. However, vascular dementia is sometimes associated with $\mathrm{AD}$ or other neurodegenerative pathology, and these patients should be examined with FDG-PET to determine the comorbidity of $\mathrm{AD}$ or other neurodegenerative pathology. Amyloid imaging can be used to remove the comorbidity of $\mathrm{AD}$ pathology when there is no amyloid deposit.

\section{Tau Imaging}

After amyloid plaques, intracellular neurofibrillary tangles are an important hallmark of AD. Neurofibrillary tangles are preferentially located in the hippocampus and associative cortical regions. ${ }^{18}$ F-2-(1-\{6-[(2-[F]Fluoroethyl)(methyl)amino]-2-naphthyl $\}$ ethylidene)malononitrile or FDDNP was initially developed for $\mathrm{A} \beta$ imaging, though this ligand binds to both $\mathrm{A} \beta$ plaques and neurofibrillary tangles in the brain. As such, FDDNP was expected to demonstrate both amyloid and neurofibrillary tangle deposits ${ }^{61}$ but has limited value for accurate measurement of $\tau$-related pathology in patients with AD. ${ }^{18} \mathrm{~F}-\mathrm{THK}-523$ was developed as a potential in vivo imaging probe for $\tau$ pathology and was reported to accumulate in regions containing a high attenuation of $\tau$ protein deposits. ${ }^{62}$ This tracer is now being used in patients with AD. Some forms of FTD, PSP, and CBD are also characterized by pathologic accumulation of $\tau$ protein. Thus, $\tau$ imaging will be useful in distinguishing these diseases from $\mathrm{AD}$.

\section{Cost-Effectiveness of PET Imaging for Dementia}

Silverman et $\mathrm{al}^{63}$ first evaluated the cost and benefit of the diagnosis of early AD by using FDG-PET and reported that appropriate use of FDG-PET can add valuable information to the clinical work-up, without adding to the overall costs of evaluation and management in the United States. In Europe, Moulin-Romsee et $\mathrm{al}^{64}$ reported that the introduction of FDG-PET into the clinical work-up of patients with suspected AD can result in substantial benefit in terms of health care cost savings and in benefit for the patient. For the present, there is still no radical treatment for $\mathrm{AD}$ because diagnosis of early or preclinical dementia remains controversial. Multicenter cohort studies should be performed to evaluate the cost-effectiveness of new biomarkers such as amyloid PET for early diagnosis of $\mathrm{AD}$ and other neurodegenerative dementias and to establish appropriate PET use criteria for the benefit of patients.

\section{CONCLUSIONS}

The use of PET enables the early detection of pathophysiologic changes in patients with dementia and allows differential diagnosis among neurodegenerative dementias. A summary of FDGPET and amyloid PET findings for each neurodegenerative dementia is shown in Table 2. Each disease has characteristic regional metabolic reduction patterns on FDG-PET. PET is now used as an in vivo imaging technique for examining neurodegenerative dementia including $\mathrm{AD}$ and for detecting the early symptomatic and preclinical stages of dementia.

\section{ACKNOWLEDGMENTS}

I thank Takamichi Murakami, MD, PhD, Chisa Hosokawa, MD, $\mathrm{PhD}$, Tomoko Hyodo, MD, PhD, Kenta Sakaguchi, RT, Kimio Usamai, RT, Yoshiyuki Nakayama, Kenji Shimamoto, PhD, and Yuzuru Yamazoe, PhD, for their support for brain FDG-PET and PiB-PET at Kinki University and former staff members at the Hyogo Institute for Aging Brain and Cognitive Disorders.

Disclosures: Kazunari Ishii—RELATED: Grant:JSPS KAKENHI Grant Number 50534103 , and 21st century research and development incentive wages at Kinki University.

\section{REFERENCES}

1. Ishii K. Clinical application of positron emission tomography for diagnosis of dementia. Ann Nucl Med 2002;16:515-25

2. McKhann GM, Knopman DS, Chertkow H, et al. The diagnosis of dementia due to Alzheimer's disease: recommendations from the National Institute on Aging-Alzheimer's Association workgroups on diagnostic guidelines for Alzheimer's disease. Alzheimers Dement 2011;7:263-69

3. Albert MS, DeKosky ST, Dickson D, et al. The diagnosis of mild cognitive impairment due to Alzheimer's disease: recommendations from the National Institute on Aging-Alzheimer's Association workgroups on diagnostic guidelines for Alzheimer's disease. Alzheimers Dement 2011;7:270-79 
4. McKhann G, Drachman D, Folstein M, et al. Clinical diagnosis of Alzheimer's disease: report of the NINCDS-ADRDA Work Group under the auspices of Department of Health and Human Services Task Force on Alzheimer's Disease. Neurology 1984;34:939-44

5. Ishii K, Sasaki M, Yamaji S, et al. Relatively preserved hippocampal glucose metabolism in mild Alzheimer's disease. Dement Geriatr Cogn Disord 1998;9:317-22

6. Maldjian JA, Whitlow CT. Whither the hippocampus? FDG-PET hippocampal hypometabolism in Alzheimer disease revisited. AJNR Am J Neuroradiol 2012;33:1975-82

7. Ishii K, Soma T, Kono AK, et al. Comparison of regional brain volume and glucose metabolism between patients with mild dementia with Lewy bodies and those with mild Alzheimer's disease. $\mathrm{J} \mathrm{Nucl}$ Med 2007;48:704-11

8. Mosconi L, Tsui WH, De Santi S, et al. Reduced hippocampal metabolism in MCI and AD: automated FDG-PET image analysis. Neurology 2005;64:1860-67

9. Karow DS, McEvoy LK, Fennema-Notestine C, et al. Relative capability of MR imaging and FDG PET to depict changes associated with prodromal and early Alzheimer disease. Radiology 2010;256:932-42

10. Kawachi T, Ishii K, Sakamoto S, et al. Comparison of the diagnostic performance of FDG-PET and VBM-MRI in very mild Alzheimer's disease. Eur J Nucl Med Mol Imaging 2006;33:801-09

11. Matsunari I, Samuraki M, Chen WP, et al. Comparison of ${ }^{18}$ F-FDG PET and optimized voxel-based morphometry for detection of Alzheimer's disease: aging effect on diagnostic performance. $\mathrm{J} \mathrm{Nucl}$ Med 2007;48:1961-70

12. Yuan Y, Gu ZX, Wei WS. Fluorodeoxyglucose-positron-emission tomography, single-photon emission tomography, and structural MR imaging for prediction of rapid conversion to Alzheimer disease in patients with mild cognitive impairment: a meta-analysis. AJNR Am J Neuroradiol 2009;30:404-10

13. Shaffer JL, Petrella JR, Sheldon FC, et al. Predicting cognitive decline in subjects at risk for Alzheimer disease by using combined cerebrospinal fluid, MR imaging, and PET biomarkers. Radiology 2013;266:583-91

14. Choo IH, Ni R, Scholl M, et al. Combination of ${ }^{18}$ F-FDG PET and cerebrospinal fluid biomarkers as a better predictor of the progression to Alzheimer's disease in mild cognitive impairment patients. $J$ Alzheimers Dis 2013;33:929-39

15. Westman E, Muehlboeck JS, Simmons A. Combining MRI and CSF measures for classification of Alzheimer's disease and prediction of mild cognitive impairment conversion. Neuroimage 2012;62:229-38

16. Zhang D, Shen D. Predicting future clinical changes of MCI patients using longitudinal and multimodal biomarkers. PLoS One 2012;7:e33182

17. Hirono N, Hashimoto M, Yasuda M, et al. The effect of APOE epsilon4 allele on cerebral glucose metabolism in $\mathrm{AD}$ is a function of age at onset. Neurology 2002;58:743-50

18. Drzezga A, Riemenschneider M, Strassner B, et al. Cerebral glucose metabolism in patients with AD and different APOE genotypes. Neurology 2005;64:102-07

19. Ossenkoppele R, van der Flier WM, Zwan MD, et al. Differential effect of APOE genotype on amyloid load and glucose metabolism in AD dementia. Neurology 2013;80:359-65

20. Minoshima S, Koeppe RA, Frey KA, et al. Stereotactic PET atlas of the human brain: aid for visual interpretation of functional brain images. J Nucl Med 1994;35:949-54

21. Minoshima S, Frey KA, Koeppe RA, et al. A diagnostic approach in Alzheimer's disease using three-dimensional stereotactic surface projections of fluorine-18-FDG PET. J Nucl Med 1995;36:1238-48

22. Herholz K, Salmon E, Perani D, et al. Discrimination between Alzheimer dementia and controls by automated analysis of multicenter FDG PET. Neuroimage 2002;17:302-16

23. Ishii K, Kono AK, Sasaki H, et al. Fully automatic diagnostic system for early- and late-onset mild Alzheimer's disease using FDG PET and 3D-SSP. Eur J Nucl Med Mol Imaging 2006;33:575-83

24. Kono AK, Ishii K, Sofue K, et al. Fully automatic differential diagnosis system for dementia with Lewy bodies and Alzheimer's disease using FDG-PET and 3D-SSP. Eur J Nucl Med Mol Imaging 2007:34:1490-97

25. Uemura T, Ishii K, Miyamoto N, et al. Computer-assisted system for diagnosis of Alzheimer disease using data base-independent estimation and fluorodeoxyglucose-positron-emission tomography and 3D-stereotactic surface projection. AJNR Am J Neuroradiol 2011;32:556-59

26. Mevel K, Desgranges B, Baron JC, et al. Detecting hippocampal hypometabolism in mild cognitive impairment using automatic voxel-based approaches. Neuroimage 2007;37:18-25

27. Klunk WE, Engler H, Nordberg A, et al. Imaging brain amyloid in Alzheimer's disease with Pittsburgh Compound-B. Ann Neurol 2004;55:306-19

28. Kudo Y, Okamura N, Furumoto S, et al. 2-(2-[2-Dimethylaminothiazol5-yl] ethenyl)-6-(2-[fluoro]ethoxy)benzoxazole: a novel PET agent for in vivo detection of dense amyloid plaques in Alzheimer's disease patients. J Nucl Med 2007;48:553-61

29. Lucignani G. Clinical applications of PET amyloid imaging: an update. Eur J Nucl Med Mol Imaging 2009;36:1185-90

30. Choi SR, Golding G, Zhuang Z, et al. Preclinical properties of ${ }^{18} \mathrm{~F}-$ AV-45: a PET agent for Abeta plaques in the brain. J Nucl Med 2009;50:1887-94

31. Cselényi Z, Jonhagen ME, Forsberg A, et al. Clinical validation of ${ }^{18} \mathrm{~F}-\mathrm{AZD} 4694$, an amyloid-beta-specific PET radioligand. $\mathrm{J} \mathrm{Nucl}$ Med 2012;53:415-24

32. Jack CR Jr, Knopman DS, Jagust WJ, et al. Hypothetical model of dynamic biomarkers of the Alzheimer's pathological cascade. Lancet Neurol 2010;9:119-28

33. Rabinovici GD, Furst AJ, Alkalay A, et al. Increased metabolic vulnerability in early-onset Alzheimer's disease is not related to amyloid burden. Brain 2010;133:512-28

34. Jack CR Jr, Wiste HJ, Vemuri P, et al. Brain beta-amyloid measures and magnetic resonance imaging atrophy both predict time-toprogression from mild cognitive impairment to Alzheimer's disease. Brain 2010;133:3336-48

35. Johnson KA, Minoshima S, Bohnen NI, et al. Appropriate use criteria for amyloid PET: a report of the Amyloid Imaging Task Force, the Society of Nuclear Medicine and Molecular Imaging, and the Alzheimer's Association. J Nucl Med 2013;54:476-90

36. Johnson KA, Minoshima S, Bohnen NI, et al. Appropriate use criteria for amyloid PET: a report of the Amyloid Imaging Task Force, the Society of Nuclear Medicine and Molecular Imaging, and the Alzheimer's Association. Alzheimers Dement 2013;9:e-1-16

37. McKeith IG, Dickson DW, Lowe J, et al. Diagnosis and management of dementia with Lewy bodies: third report of the DLB Consortium. Neurology 2005;65:1863-72

38. Albin RL, Minoshima S, D'Amato CJ, et al. Fluoro-deoxyglucose positron emission tomography in diffuse Lewy body disease. $\mathrm{Neu}$ rology 1996;47:462-66

39. Ishii K, Imamura T, Sasaki M, et al. Regional cerebral glucose metabolism in dementia with Lewy bodies and Alzheimer's disease. Neurology 1998;51:125-30

40. Bohnen NI, Koeppe RA, Minoshima S, et al. Cerebral glucose metabolic features of Parkinson disease and incident dementia: longitudinal study. J Nucl Med 2011;52:848-55

41. McKeith I, O'Brien J, Walker Z, et al. Sensitivity and specificity of dopamine transporter imaging with ${ }^{123}$ I-FP-CIT SPECT in dementia with Lewy bodies: a phase III, multicentre study. Lancet Neurol 2007;6:305-13

42. Treglia G, Cason E. Diagnostic performance of myocardial innervation imaging using MIBG scintigraphy in differential diagnosis between dementia with Lewy bodies and other dementias: a systematic review and a meta-analysis. J Neuroimaging 2012;22:111-17

43. Shimada $\mathrm{H}$, Shinotoh $\mathrm{H}$, Hirano S, et al. Beta-amyloid in Lewy body 
disease is related to Alzheimer's disease-like atrophy. Mov Disord 2013;28:169-75

44. Gomperts SN, Rentz DM, Moran E, et al. Imaging amyloid deposition in Lewy body diseases. Neurology 2008;71:903-10

45. Gomperts SN, Locascio JJ, Marquie M, et al. Brain amyloid and cognition in Lewy body diseases. Mov Disord 2012;27:965-73

46. Fujishiro H, Iseki E, Higashi S, et al. Distribution of cerebral amyloid deposition and its relevance to clinical phenotype in Lewy body dementia. Neurosci Lett 2010;486:19-23

47. Clinical and neuropathological criteria for frontotemporal dementia; the Lund and Manchester Groups. J Neurol Neurosurg Psychiatry 1994;57:416-18

48. Ishii K, Sakamoto S, Sasaki M, et al. Cerebral glucose metabolism in patients with frontotemporal dementia. J Nucl Med 1998;39:1875-78

49. Kanda T, Ishii K, Uemura T, et al. Comparison of grey matter and metabolic reductions in frontotemporal dementia using FDG-PET and voxel-based morphometric MR studies. Eur J Nucl Med Mol Imaging 2008;35:2227-34

50. Engler $\mathrm{H}$, Santillo AF, Wang SX, et al. In vivo amyloid imaging with PET in frontotemporal dementia. Eur J Nucl Med Mol Imaging 2008;35:100-06

51. Rabinovici GD, Furst AJ, O’Neil JP, et al. ${ }^{11}$ C-PIB PET imaging in Alzheimer disease and frontotemporal lobar degeneration. Neurology 2007;68:1205-12

52. Juh R, Pae CU, Kim TS, et al. Cerebral glucose metabolism in corticobasal degeneration comparison with progressive supranuclear palsy using statistical mapping analysis. Neurosci Lett 2005;383:22-27

53. Juh R, Kim J, Moon D, et al. Different metabolic patterns analysis of Parkinsonism on the 18F-FDG PET. Eur J Radiol 2004;51:223-33

54. Klein RC, de Jong BM, de Vries JJ, et al. Direct comparison between regional cerebral metabolism in progressive supranuclear palsy and Parkinson's disease. Mov Disord 2005;20:1021-30

55. Hosaka K, Ishii K, Sakamoto S, et al. Voxel-based comparison of regional cerebral glucose metabolism between PSP and corticobasal degeneration. J Neurol Sci 2002;199:67-71

56. Hirono N, Ishii K, Sasaki M, et al. Features of regional cerebral glucose metabolism abnormality in corticobasal degeneration. Dement Geriatr Cogn Disord 2000;11:139-46

57. Hashimoto M, Ishikawa M, Mori E, et al. Diagnosis of idiopathic normal pressure hydrocephalus is supported by MRI-based scheme: a prospective cohort study. Cerebrospinal Fluid Res 2010;7:18
58. Jagust WJ, Friedland RP, Budinger TF. Positron emission tomography with $\left[{ }^{18} \mathrm{~F}\right]$ fluorodeoxyglucose differentiates normal pressure hydrocephalus from Alzheimer-type dementia. J Neurol Neurosurg Psychiatry 1985;48:1091-96

59. Calcagni ML, Lavalle M, Mangiola A, et al. Early evaluation of cerebral metabolic rate of glucose (CMRglu) with ${ }^{18} \mathrm{~F}-\mathrm{FDG}$ PET/CT and clinical assessment in idiopathic normal pressure hydrocephalus (INPH) patients before and after ventricular shunt placement: preliminary experience. Eur J Nucl Med Mol Imaging 2012;39:236-41

60. Rinne JO, Wong DF, Wolk DA, et al. $\left[{ }^{18} \mathrm{~F}\right]$ Flutemetamol PET imaging and cortical biopsy histopathology for fibrillar amyloid beta detection in living subjects with normal pressure hydrocephalus: pooled analysis of four studies. Acta Neuropathol 2012;124:833-45

61. Shin J, Kepe V, Barrio JR, et al. The merits of FDDNP-PET imaging in Alzheimer's disease. J Alzheimers Dis 2011;26(suppl 3):135-45

62. Fodero-Tavoletti MT, Okamura N, Furumoto S, et al. ${ }^{18}$ F-THK523: a novel in vivo tau imaging ligand for Alzheimer's disease. Brain 2011;134:1089-100

63. Silverman DH, Gambhir SS, Huang HW, et al. Evaluating early dementia with and without assessment of regional cerebral metabolism by PET: a comparison of predicted costs and benefits. J Nucl Med 2002;43:253-66

64. Moulin-Romsee G, Maes A, Silverman D, et al. Cost-effectiveness of ${ }^{18} \mathrm{~F}$-fluorodeoxyglucose positron emission tomography in the assessment of early dementia from a Belgian and European perspective. Eur J Neurol 2005;12:254-63

65. Mathis CA, Wang Y, Holt DP, et al. Synthesis and evaluation of ${ }^{11} \mathrm{C}$-labeled 6-substituted 2-arylbenzothiazoles as amyloid imaging agents. J Med Chem 2003;46:2740-54

66. Nyberg S, Jonhagen ME, Cselenyi Z, et al. Detection of amyloid in Alzheimer's disease with positron emission tomography using $\left[{ }^{11}\right.$ C]AZD2184. Eur J Nucl Med Mol Imaging 2009;36:1859-63

67. Agdeppa ED, Kepe V, Liu J, et al. Binding characteristics of radiofluorinated 6-dialkylamino-2-naphthylethylidene derivatives as positron emission tomography imaging probes for beta-amyloid plaques in Alzheimer's disease. J Neurosci 2001;21:RC189

68. Nelissen N, Van Laere K, Thurfjell L, et al. Phase 1 study of the Pittsburgh compound B derivative ${ }^{18} \mathrm{~F}$-flutemetamol in healthy volunteers and patients with probable Alzheimer disease. J Nucl Med 2009;50:1251-59

69. Rowe CC, Ackerman U, Browne W, et al. Imaging of amyloid beta in Alzheimer's disease with ${ }^{18} \mathrm{~F}-\mathrm{BAY} 94-9172$, a novel PET tracer: proof of mechanism. Lancet Neurol 2008;7:129-35 